\title{
Plasma Structuring in the Polar Cap
}

\author{
Sunanda BASU ${ }^{1}$, Santimay BASU ${ }^{2}$, E. J. WEBER ${ }^{2}$, and G. J. BISHOP ${ }^{2}$ \\ ${ }^{1}$ Institute for Space Research, Boston College, Newton Center, MA 02159, U.S.A. \\ ${ }^{2}$ Geophysical Laboratory (LIS), Hanscom AFB, MA 01731, U.S.A.
}

(Received February 19, 1990; Accepted March 8, 1990)

\begin{abstract}
Propagation experiments providing scintillation, total electron content and drift data in the field of view of an all-sky imager near the magnetic pole in Greenland are utilized to investigate the manner in which ionospheric plasma becomes structured within the polar cap. It is found that under IMF $B_{z}$ southward conditions, large scale ionization patches which are convected through the dayside cusp into the polar cap get continually structured. The structuring occurs through the $\boldsymbol{E} \times \boldsymbol{B}$ gradient drift instability process which operates through an interaction between the antisunward plasma convection in the neutral rest frame and large scale plasma density gradients that exist at the edges of the ionization patches. It is shown that with the increase of solar activity the strength of the irregularities integrated through the ionosphere is greatly increased. Under the IMF $B_{z}$ northward conditions, the plasma structuring occurs around the polar cap arcs in the presence of inhomogeneous electric field or disordered plasma convection. In that case, the irregularity generation is caused by the competing processes of non-linear KelvinHelmholtz instability driven by sheared plasma flows and the gradient drift instability process which operates in the presence of dawn-dusk motion of arc structures. The integrated strength of this class of irregularities also exhibits marked increase with increasing solar activity presumably because the ambient plasma density over the polar cap is enhanced.
\end{abstract}

\section{Introduction}

Plasma subject to stress can structure if the stress-driven instability growth-rates exceed the rates of structure dissipation over a sufficiently large number of instability growth-times. At ionospheric $E$-region altitudes relatively short chemical time constants (photo-chemical lifetimes of minutes) tend to tie structures to irregularity sources. $F$ region irregularities are quite complex because their lifetimes could be several hours depending on their scale size (VICKREY and KELLEY, 1982). Over hours polar $F$-region plasma can move thousands of kilometers. Thus its instantaneous structure represents the cumulative consequence of competing variable growth and dissipative processes encountered over the history of its transpolar and/or return flow trajectory. It is thus not surprising that we are at present a long way from confidence in our ability to physically model or even specify the dominant processes controlling high latitude ionospheric plasma structuring.

It is for this reason that a working group has been set-up under the U.S. National Science Foundation's "Coupling, Energetics and Dynamics of Atmospheric Regions (CEDAR)" initiative to study high latitude plasma structures (HLPS). HLPS is an ambitious program to address the goal of understanding the processes controlling source, 
evolution and decay of ionospheric structures using a transpolar array of instrumentation defining key physical parameters. The work of the HLPS group has been primarily organized around the finding that the large scale structuring ( $\sim$ hundreds of $\mathrm{km})$ in the very high latitude ionosphere-magnetosphere (I-M) system is controlled by the northsouth component, namely, $B_{z}$ of the IMF. Using sensitive all-sky imaging photometers (ASIPs) located deep within the dark winter polar cap, it has been shown that when IMF $B_{z}$ is negative, large $(\sim 1000 \mathrm{~km})$ regions of convecting density enhancement known as patches are observed, whereas, when IMF $B_{z}$ is positive, sun-aligned arcs are found to populate the polar cap (WEBER et al., 1984; BUCHAU et al., 1985). That both patches and arcs are associated with small-scale density and electric field turbulence ( $\sim \mathrm{kms}$ to $\mathrm{m}$ ) has been determined by a variety of in situ and remote sensing techniques (CARLSON et al., 1988a).

The first multi-technique HLPS campaign of February 1988 offered a unique opportunity to further investigate the structuring of patches and arcs. Coordinated measurements carried out with the Sondrestrom incoherent scatter radar, all-sky imaging photometers (ASIPs), orbiting and quasi-stationary satellites and ionosondes allowed us to present a study of these two distinct classes of large scale plasma structures and associated turbulence in the polar cap. These results obtained under low sunspot conditions $(\mathrm{SSN}=40)$ have already been published (BASU et al., 1989). A similar data base was utilized to investigate plasma structuring over the polar cap during the second HLPS campaign in December 1988 when the sunspot number was as high as 180 . During this campaign, spaced receiver scintillation measurements could be performed to obtain drift and anisotropy of the plasma density irregularities. In this paper the nature of irregularity structures in polar cap arcs and patches observed during the second HLPS campaign under high sunspot conditions will be discussed and contrasted with the results of the first HLPS campaign which as previously mentioned was performed under low sunspot conditions.

\section{Results}

A polar cap patch and an arc event are studied in detail here and contrasted with the February events published by BASU et al. (1989). The patch event was observed on December 6, 1988, while the arc event was observed on December 12, 1988.

\subsection{Polar cap patch}

The patches were identified on the imager located at Qaanaaq, Greenland between 00-02 UT on December 6, 1988. This period corresponded to extremely low magnetic activity with $\Sigma K p=7^{+}$and $6^{+}$for December 5 and 6 , respectively. In fact, the period December 5-7 constituted the three quietest days of the month. Yet polar cap patches were observed contrary to generally held opinion that patches are observed under moderately high and variable $K p$ conditions (ANDERSON et al., 1988). No IMF data was available at this time but $B_{z}$ was predominantly southward ( -2 to $-3 \mathrm{nT}$ ) between 21-23 UT of December 5, 1988 and between 02:30-05:30 UT on December 6 and $B_{y}$ was positive for 22 hours before and 18 hours after the data gap. Given the experimental evidence for the existence of patches during the IMF data gap, it seems fairly reasonable to assume that $B_{z}$ remained southward throughout the period.

Figure 1 shows the geometry of the observations. At Qaanaaq (QA), an all-sky 


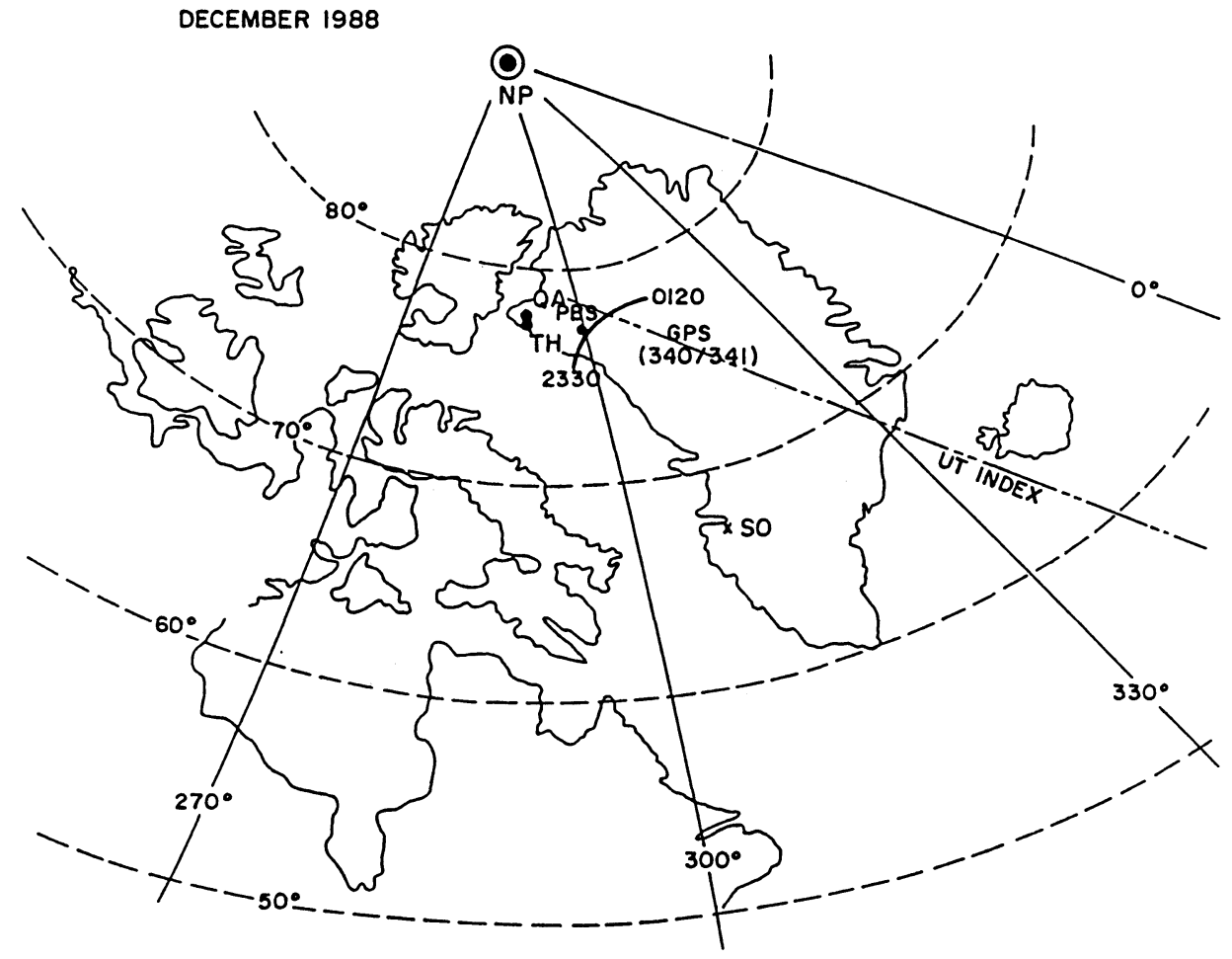

Fig. 1. The geometry of the scintillation and TEC observations in Greenland on December 6, 1988.

imager was operated, while at Thule (TH), polar beacon satellite signals at $244 \mathrm{MHz}$ were acquired by a spaced receiver system which provided amplitude and phase scintillations, as well as, irregularity drift velocity. The intersection of the propagation path with an ionospheric height of $350 \mathrm{~km}$ is indicated by a dot $(\cdot)$ and identified by PBS. The phase coherent signals at 1.2 and $1.6 \mathrm{GHz}$ transmitted by the Global Positioning Satellite (GPS) were also monitored at Thule to obtain the total electron content (TEC) of the ionosphere. The locus of the $350 \mathrm{~km}$ ionosphere intersection with the ray path for this satellite between 23:30 and 01:20 UT is indicated on the diagram and identified by GPS.

Figure 2 shows the results of the relative TEC obtained from the differential phase measurements with the GPS satellite. The series of increases and decreases of TEC indicates successive encounters of the propagation path with the drifting polar cap patches of ionization. The average enhancement of TEC in these patches above the neighboring minimum amount to $10 \times 10^{16} \mathrm{~m}^{-2}$. Correcting for the slant orientation of the propagation path, the equivalent vertical TEC of the ionization patches above the background is obtained as $7 \times 10^{16} \mathrm{~m}^{-2}$. This contrast of TEC between the patch and the neighboring background may be considered to be equivalent to a variation of $f_{\mathrm{o}} F_{2}$ from 7 $\mathrm{MHz}$ with a slab thickness of $200 \mathrm{~km}$ within the patch to $f_{0} F_{2}$ of $5 \mathrm{MHz}$ in the background with a slab thickness of $150 \mathrm{~km}$.

The time history of irregularities of several $\mathrm{km}$ to several tens of $\mathrm{m}$ as observed by $250 \mathrm{MHz}$ phase and amplitude scintillation at the ionospheric intersection point PBS in 


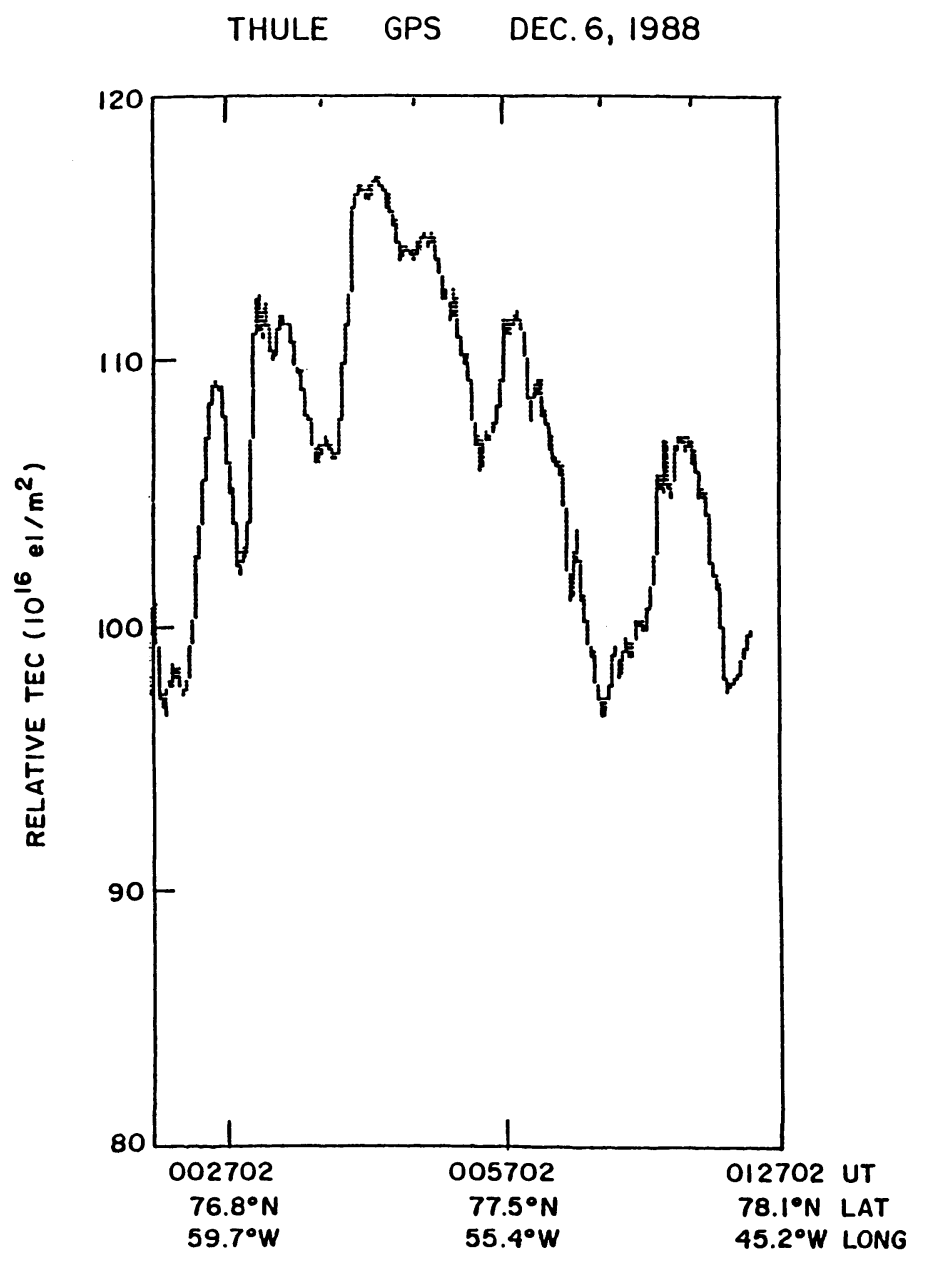

Fig. 2. TEC observations using a GPS satellite from Thule during a polar cap patch event on December 6, 1988.

Fig. 1 which occur in association with the larger scale TEC enhancements is shown in Fig. 3. Large peaks with $S_{4}=0.8$ are observed in conjunction with phase scintillations exceeding 10 radians (for $82-\mathrm{sec}$ detrend intervals). The other interesting feature of the data is that the decorrelation interval (for 50 percent decorrelation) is fairly constant with time varying at most by a factor of 2 from 0.2 to $0.4 \mathrm{sec}$.

The scintillation data were being taken with three spaced antennas defining approximately an isosceles right-triangle with smaller sides of approximately $170 \mathrm{~m}$, and using a slightly modified version of the FEDOR (1967) technique as discussed at length by COSTA et al. (1988) in their Subsection 4.1, we obtain the direction and magnitude of the vector drift as given in Fig. 4. The magnitude of the drift generally varies between 0.4-0.8 $\mathrm{km} \mathrm{s}^{-1}$. The direction of the drift varies between $90-140^{\circ}$ with this range of directions indicating drifts varying between magnetic eastward to southeastward (i.e., approximately 
THULE POLAR BEACON $250 \mathrm{MHz}$ DEC. 6, 1988
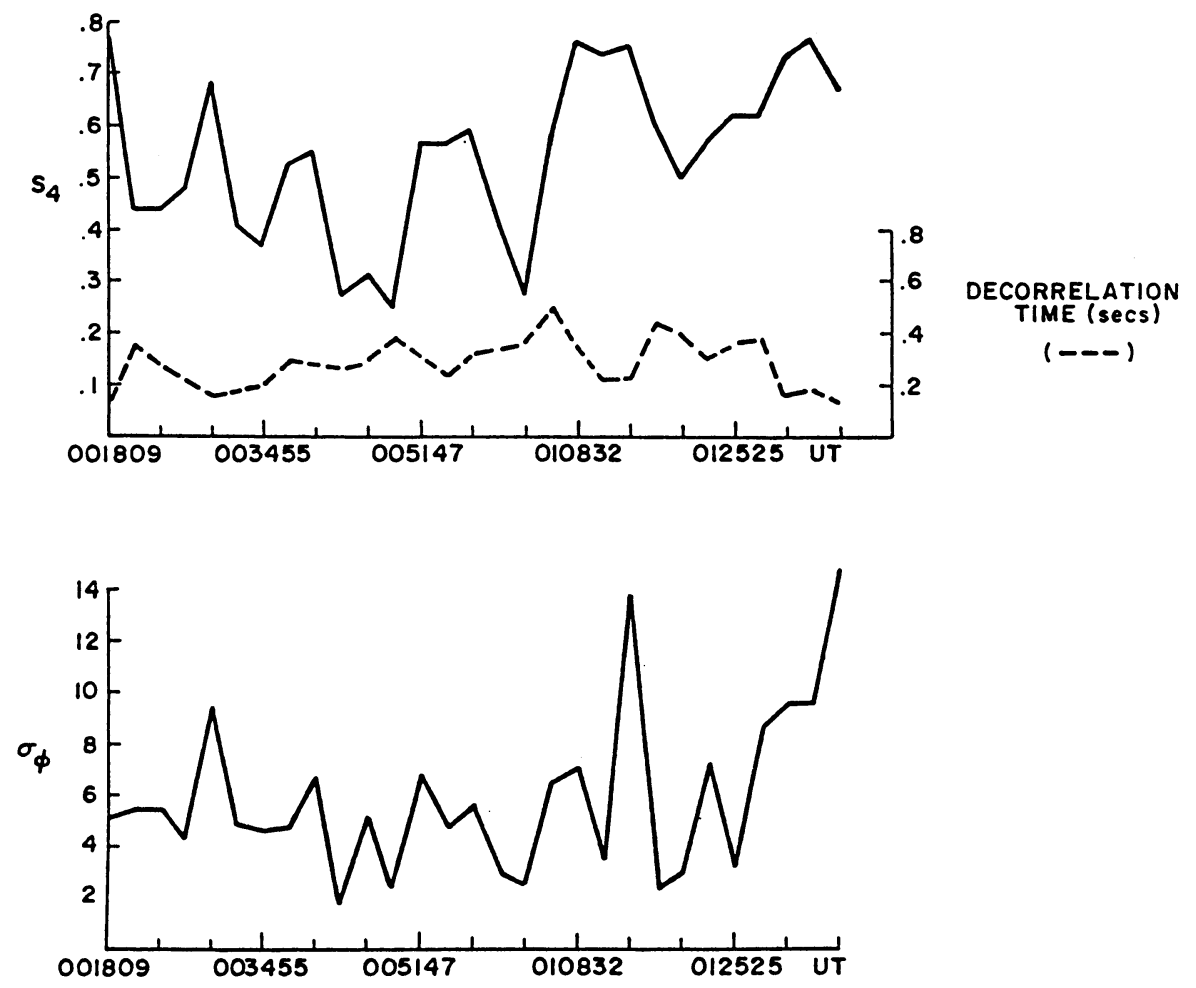

Fig. 3. The same as Fig. 2 for phase and intensity scintillations and decorrelation time at $250 \mathrm{MHz}$. The phase scintillation is measured in radians, while the intensity scintillation is expressed in terms of the $S_{4}$ index, i.e., the second central moment of the intensity.

antisunward), as shown on the corrected geomagnetic latitude (CGL) and time (CGLT) polar plot in Fig. 5.

Neutral wind measurements were available from Thule where the University of Michigan personnel were operating their Fabry-Perot interferometer. Between 00-02 UT the average antisunward drift was $300 \mathrm{~ms}^{-1}$ (J. P. Thayer, private communication). It is possible to estimate the time required by the trailing edges of convecting patches to develop small-scale structures as they drift antisunward in the rest frame of the neutrals. The zero-order growth time is given by $L_{\mathrm{N}} /\left(V_{\mathrm{d}}-U_{\mathrm{N}}\right)$ where $L_{\mathrm{N}}$ is the density gradient scale length and $V_{\mathrm{d}}$ and $U_{\mathrm{N}}$ are the plasma drift and neutral wind velocities, respectively. Now $L_{N}$ may be estimated by converting the temporal variations of vertical TEC at the trailing edges of patches to spatial gradients by utilizing the plasma drift velocity. Using this method one gets $L_{\mathrm{N}}$ values as small as $40 \mathrm{~km}$. This when combined with the average plasma drift of approximately $600 \mathrm{~ms}^{-1}$ and the neutral wind of $300 \mathrm{~ms}^{-1}$ provides growth times on the order of 2 mins which is very short indeed. Thus we can expect the density gradients at the edges of patches to become quickly unstable and generate scintillation producing irregularities. This finding is consistent with earlier studies of patches (WEBER 


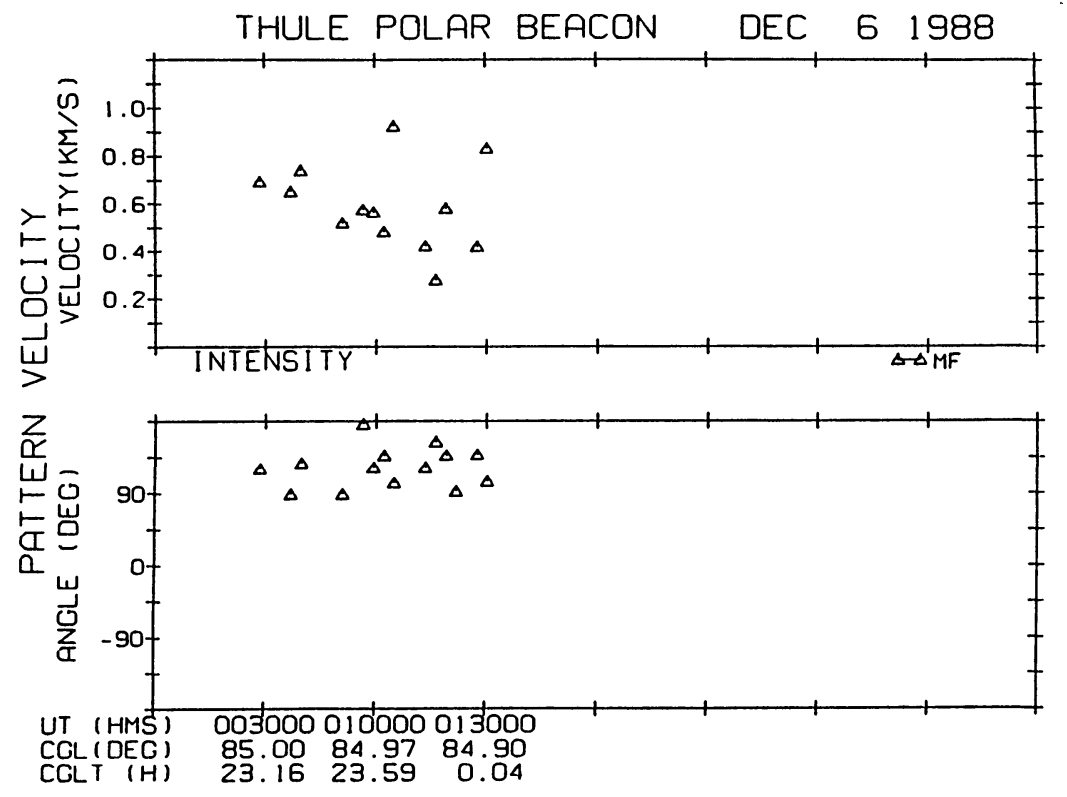

Fig. 4. The pattern drift velocity and its direction obtained for the data shown in Fig. 3 by using spaced antennas.

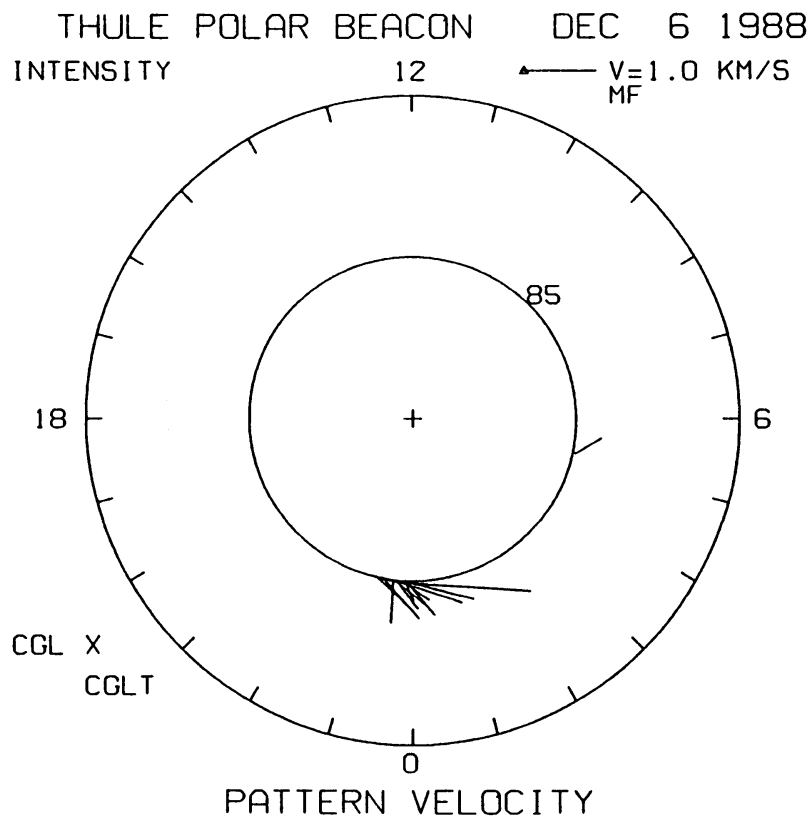

Fig. 5. The drift velocity vector for the December 6, 1988 patch event shown on a polar plot of corrected magnetic latitude and local time. 
et al., 1984, 1986; BUCHAU et al., 1985). What remains rather enigmatic is the process whereby a succession of patches can enter the polar cap under extended quiet magnetic conditions. As already mentioned, this is contrary to modeling studies performed earlier (ANDERSON et al., 1988). The cross polar cap potential which can be estimated from the DMSP drift measurements (HAIRSTON and HEELIS, 1989) during this event will be utilized in future to provide further insight into the problem.

\subsection{Polar cap arc}

The geometry of supporting measurements during the appearance of the sun-aligned arc on December 12, 1988 between 10:30-11:30 UT is shown in Fig. 6 with the symbols having the same meaning as in Fig. 1. Two images of the sun-aligned arc using the Qaanaaq ASIP at 630 and $427.8 \mathrm{~nm}$ are shown in Fig. 7. The original all-sky lens $\left(180^{\circ}\right.$ field of view) images have been transformed to geographic projections, and the polar cap is displayed as if viewed from above. In order to perform these transformations, an altitude of $250 \mathrm{~km}$ was assumed for the $630 \mathrm{~nm}$ and $110 \mathrm{~km}$ for the $427.8 \mathrm{~nm}$ emission height, respectively. Geographic latitudes from $70^{\circ}$ to $85^{\circ} \mathrm{N}$ are shown at $5^{\circ}$ intervals, and geographic longitudes from $0^{\circ}$ to $135^{\circ} \mathrm{W}$ are shown at $15^{\circ}$ intervals. The "p" and the "g" on the images are the $250(110) \mathrm{km}$ intersections of the ray paths for the $630(427.8)$ $\mathrm{nm}$ emissions from Thule to the polar beacon and to the GPS satellites. The scintillation and spaced-receiver drift measurements obtained from the polar beacon ( $p$ ) and the TEC

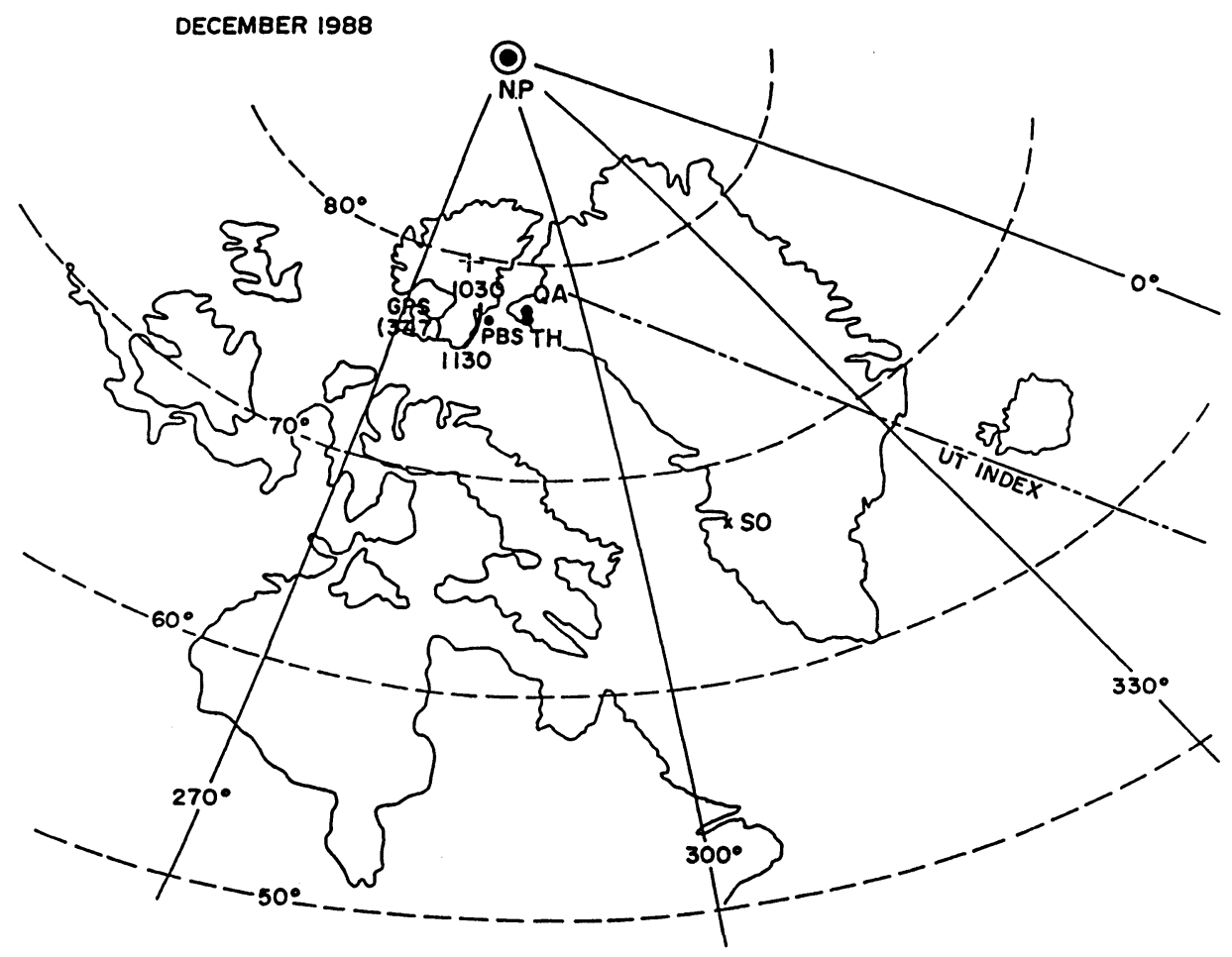

Fig. 6. The geometry of the scintillation and TEC observations in Greenland on December 12, 1988. 

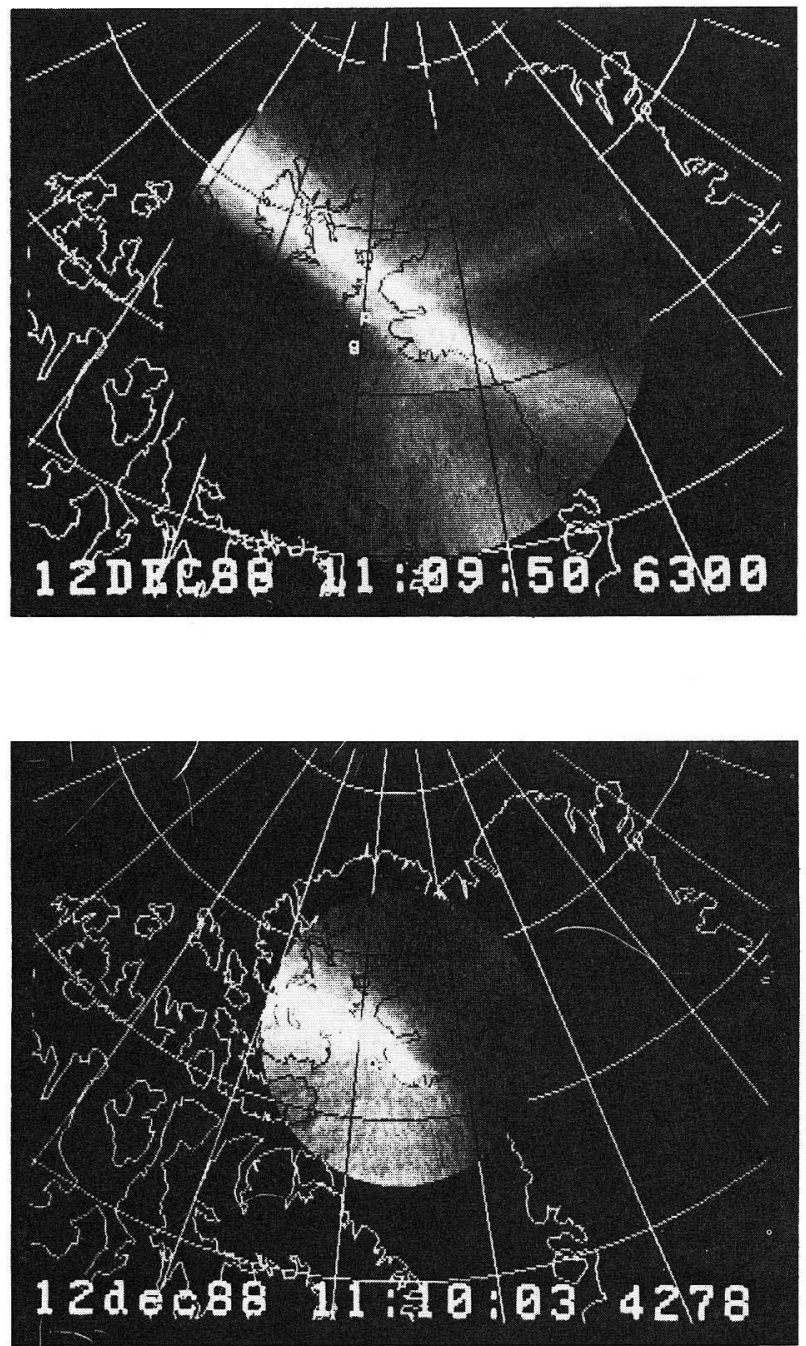

Fig. 7. Two ASIP images of the polar cap sun-aligned arc from Qaanaaq obtained on December 12, 1988 at 630 and $427.8 \mathrm{~nm}$. The images are projected to $250(110) \mathrm{km}$ for the $630(427.8) \mathrm{nm}$ images. The points $\mathrm{p}$ and $\mathrm{g}$ represent the $250(110) \mathrm{km}$ intersections of the ray path from Thule to the polar beacon and GPS satellites.

from the GPS (g) will thus provide information regarding the large and small-scale structuring of this polar cap arc. The arc is observed at Qaanaaq in the local morning hours such that its equatorward side is toward dawn and consequently its poleward side is towards dusk. The fact that both emissions are present indicates that the particle precipitation responsible for this sun-aligned arc is energetic enough to create ionization in the $E$-region in addition to the $F$-region ionization which produces the $630-\mathrm{nm}$ emission.

The TEC from GPS associated with the arc is shown in Fig. 8. The differential 
THULE GPS DEC.12, 1988

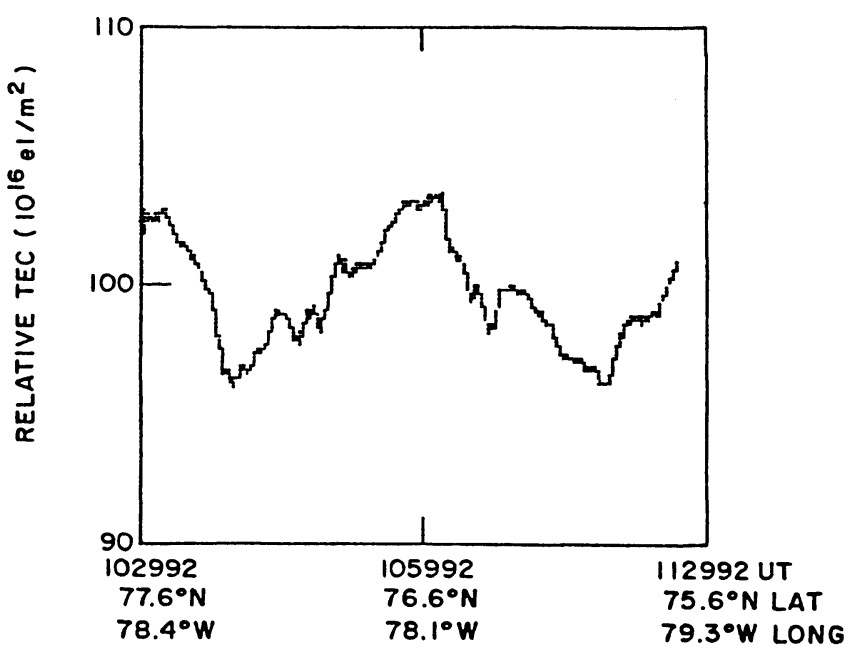

Fig. 8. TEC observations using a GPS satellite from Thule during the sun-aligned polar cap arc event on December 12, 1988.

content increase of $7 \times 10^{16} \mathrm{el} \mathrm{m}^{-2}$ along the slant path, equivalent to a vertical TEC increase of $5 \times 10^{16} \mathrm{el} \mathrm{m}^{-2}$, is less than that seen in patches but the more important difference is the rather gradual rate of increase and decrease for the central structure. This could be due to the generally smaller magnitude of the velocities perpendicular to the length of the arc as obtained from the spaced-receiver technique to be presented below. However, the TEC behavior for this sunspot maximum arc does provide a fairly dramatic contrast when compared with the sunspot minimum arc (BASU et al., 1989).

The phase and amplitude scintillations associated with the arc shown in Fig. 9 is much lower than that observed in the case of patches. There is a peak in the scintillation magnitude at approximately 11:15 UT. Of considerable interest is the variability of the decorrelation time and larger magnitude observed which (since all the scintillations are in the weak-scatter regime) indicates slower drift velocities and variation in their magnitude. The drift variability can also be gauged from the intensity scintillation spectrum obtained at 11:12:08 UT when compared with that obtained at 11:17:45 UT. Whereas the Fresnel filter frequency $(=u / \sqrt{2 \lambda z}$, where $u$ is the drift velocity perpendicular to the ray path, $\lambda$ is the radio wavelength and $z$ the distance between the screen and the receiver) is at $0.2 \mathrm{~Hz}$ in Fig. 10(a), it is pushed out to $1.5 \mathrm{~Hz}$ in Fig. 10(b). This indicates a factor of seven variation in the drift velocity.

The spaced receiver drift measurements shown in Figs. 11 and 12 provide direct measurements of the magnitude and azimuth of the drift velocity vector in cartesian and polar coordinates as already described in Figs. 4 and 5 for the case of patches. From Fig. 11 we find that just after 11:15 UT the pattern velocity direction changes from $-90^{\circ}$ azimuth to $+90^{\circ}$ and at the same time the magnitude of the velocity increases by a factor of seven. from 100 to $700 \mathrm{~ms}^{-1}$ in agreement with the information obtained from the 
THULE POLAR BEACON $250 \mathrm{MHz}$ DEC. 12,1988
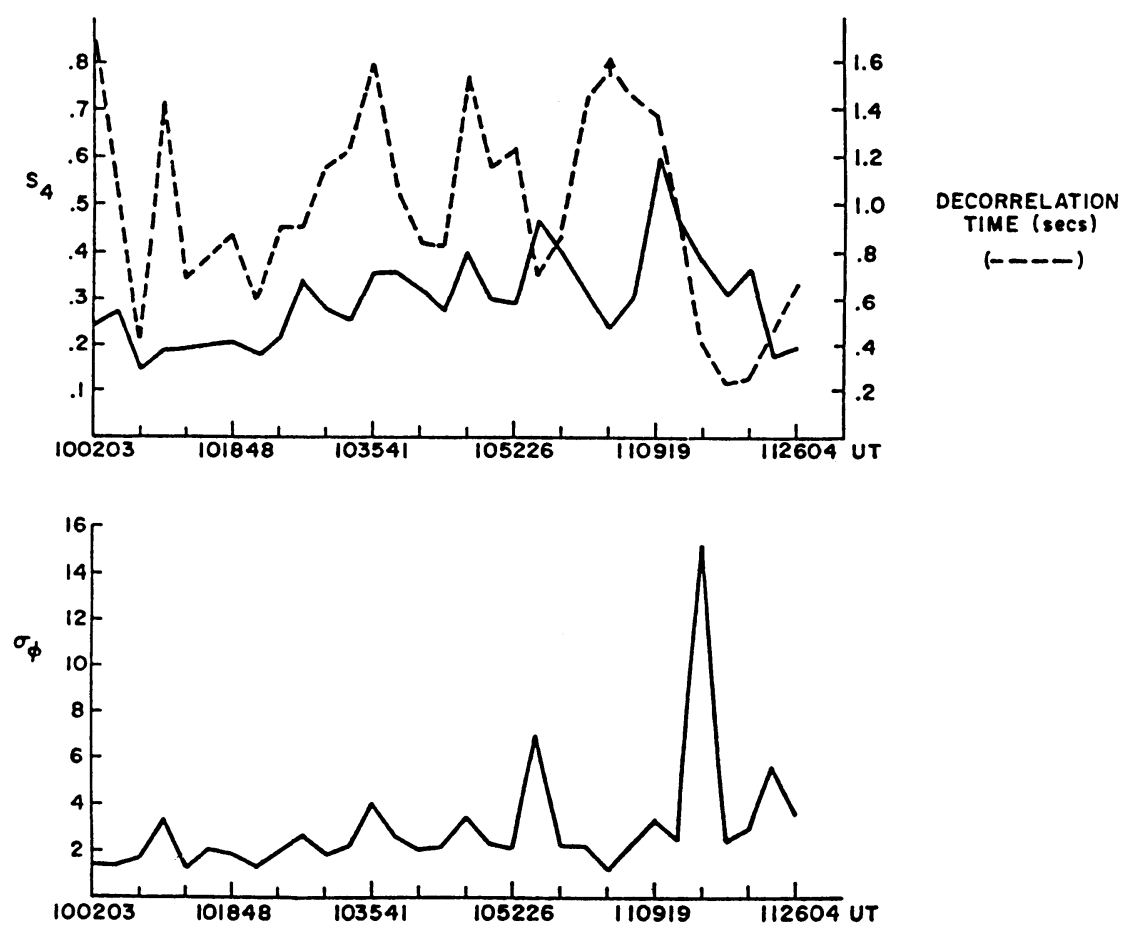

Fig. 9. Same as in Fig. 3 except for the sun-aligned polar cap arc event on December 12, 1988.

spectral comparison. It was mentioned earlier that the peak in the scintillations was at approximately 11:15 UT. From Fig. 11, we note that the scintillation peak is co-located with this velocity shear. From the polar plot in the CGL-CGMLT coordinate system in Fig. 12 we note that the shear is in a direction such that an antisunward drift seen at earlier local time reverses to a sunward drift at a later time. If the time history of the images of the type presented in Fig. 7 shows that initially the point $p$ was equatorward of the arc and at a later time it was encompassed by the arc, then this finding will be consistent with the view that sun-aligned polar cap arcs are found in a region of convergent electric fields (CARLSON et al., 1988b; VALLADARES and CARLSON, 1990). A careful comparison will be made between the position of the point $p$ and the arc boundaries in order to establish the location of the sheared flow.

\section{Comparison with Earlier Observations}

A comparison between patches obtained at various phases of the sunspot cycle provide evidence for considerable variability for TEC within patches. The first TEC measurements with GPS at Thule in February 1984 with monthly mean sunspot number of 85 provided patches with TEC varying between $10 \times 10^{16} \mathrm{el} \mathrm{m}^{-2}$ to $15 \times 10^{16} \mathrm{el} \mathrm{m}^{-2}$ (WEBER et al., 1986). The patches observed during the low sunspot conditions of 
THULE POLAR BEACON DEC. 12,1988

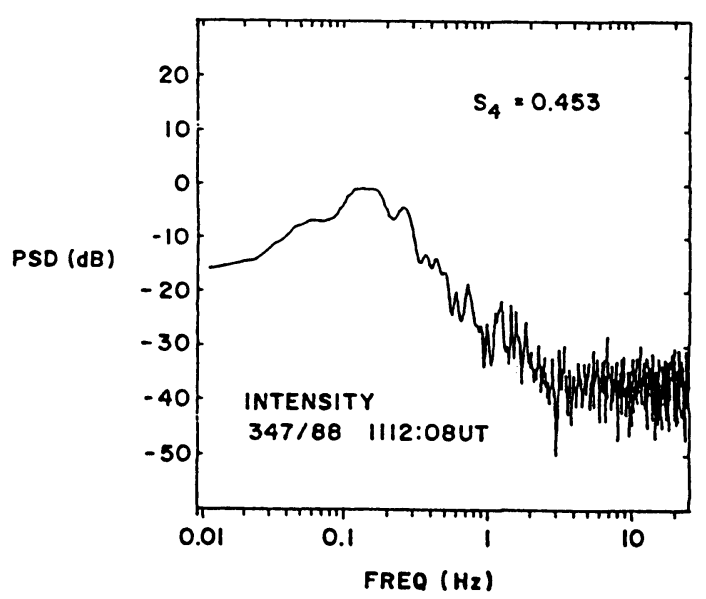

(a)

THULE POLAR BEACON DEC.12,1988

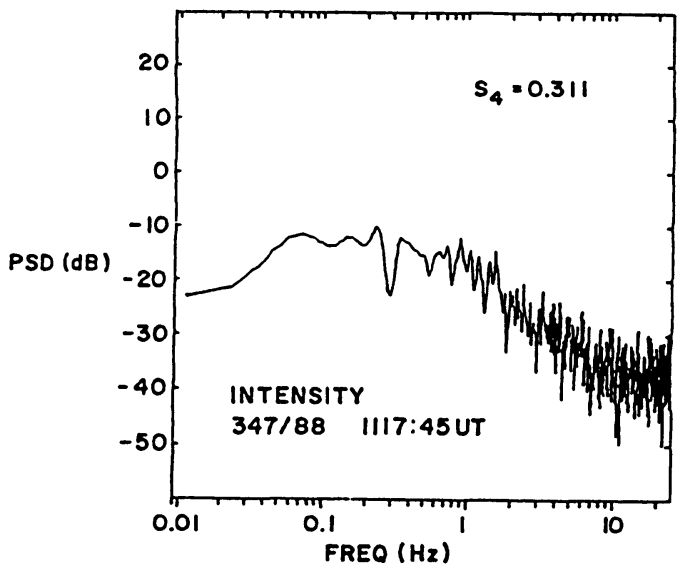

(b)

Fig. 10. (a) Intensity scintillation spectrum at 11:12:08 UT on December 12, 1988. (b) The same at 11:17:45 UT on December 12, 1988 during a large sheared flow event. 


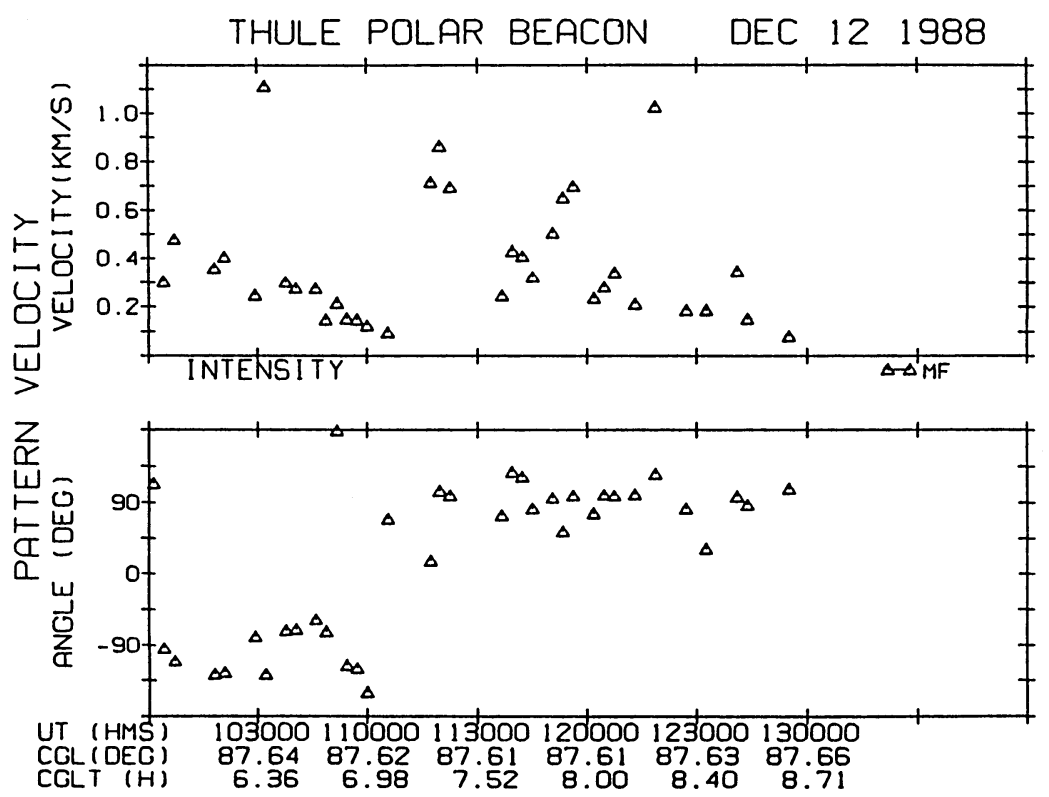

Fig. 11. Same as in Fig. 4 but for the data shown in Fig. 9. Note the velocity shear at 11:15 UT.

February 1988 (sunspot number 40) provided evidence for TEC in patches varying between 5 and $10 \times 10^{16} \mathrm{el} \mathrm{m}^{-2}$ (BASU et al., 1989) which were not unlike those found in the present case. It should be remembered though that these patches seen during this high sunspot period were observed during a time of very little magnetic activity. The mechanism whereby a succession of patches can enter the polar cap during such an extended period of magnetic quiet merits much further investigation.

The structuring of these patches by the $\boldsymbol{E} \times \boldsymbol{B}$ instability is fairly well understood (TSUNODA, 1988). However, as with the TEC structures observed in December 1988 the leading edge occasionally seems steeper than the trailing edge. In prior studies, similar situations were observed in in situ data (TSUNODA, 1988; BASU et al., 1990).

The comparison of TEC associated with polar cap arcs as a function of sunspot cycle and their structuring still remains an intriguing problem. The TEC increase was approximately 3 times larger in the high sunspot case $\left(5 \times 10^{16} \mathrm{el} \mathrm{m}^{-2}\right)$ as compared to the February 1988 arc (about $2 \times 10^{16} \mathrm{el} \mathrm{m}^{-2}$ ). Indeed many other arcs observed during the Polar Arcs Campaign of February 1987 provided similarly low values of TEC. Since we are measuring the relative TEC increase, this seems to indicate that particle precipitation during high sunspot conditions creates a much higher level of ionization. It is necessary to verify this hypothesis with modeling studies. Further, the increased scintillation coinciding with the sheared plasma flow gives further credibility to the velocity sheardriven Kelvin-Helmholtz instability as a plausible mechanism for structuring associated with polar cap arcs (BASU et al., 1988; KESKINEN et al., 1988). The weaker irregularities could be generated at the TEC gradients by the background drifts through the $\boldsymbol{E} \times \boldsymbol{B}$ process (WEBER et al., 1989). 


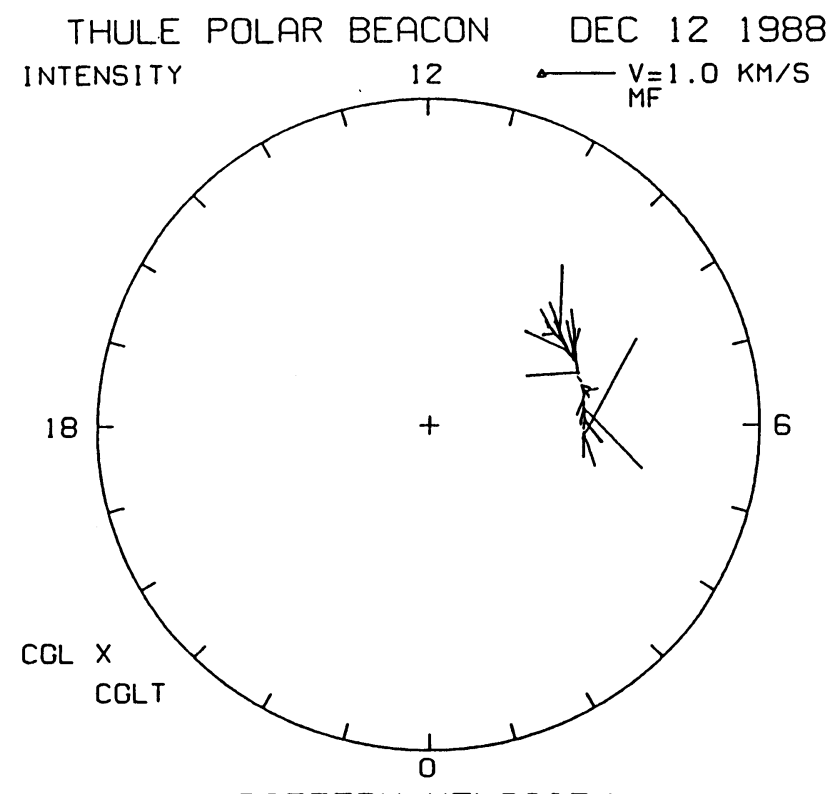

PATTERN VELOCITY

Fig. 12. Same as in Fig. 5 but for the polar cap sun-aligned arc event of December 12, 1988.

The work at Boston College was partially supported by NSF Grant ATM-8715445 and by GL Contract F19628-90-K-0007. We thank the Danish Commission for Scientific Research in Greenland for permission to conduct ground and aircraft experiments for Thule AB under Projects 28-88 and 30-88.

\section{REFEREBCES}

Anderson, D. N., J. Buchau, and R. A. Heelis, Origin of density enhancements in the winter polar cap ionosphere, Radio Sci., 23, 513, 1988.

Basu, S., S. Basu, E. Mackenzie, P. F. Fougere, W. R. Coley, N. C. Maynard, J. D. Winningham, M. Sugiura, W. B. Hanson, and W. R. HoEgY, Simultaneous density and electric field fluctuation spectra associated with velocity shears in the auroral oval, J. Geophys. Res., 93, 115, 1988.

Basu, S., S. Basu, C. E. Valladares, E. J. Weber, J. Buchau, G. J. Bishop, and B. W. Reinisch, Coordinated observations of high latitude ionospheric turbulence, SPI Conference Proceedings and Reprint Series, 8, 137, 1989.

Basu, S., S. Basu, E. Mackenzie, W. R. Coley, J. R. Sharber, and W. R. Hoegy, Plasma structuring by the gradient-drift instability at high latitudes and comparison with velocity-shear driven processes, J. Geophys. Res., 95, 1990 (in press).

Buchau, J., E. J. Weber, D. N. Anderson, H. C. Carlson, Jr., J. G. Moore, B. W. Reinisch, and R. C. LIVINGSTON, Ionospheric structures in the polar cap: Their origin and relation to $250-\mathrm{MHz}$ scintillation, Radio Sci., 20, 325-338, 1985.

Carlson, H. C., Jr., E. J. Weber, L. P. Block, and S. Basu, Satellite, airborne, and radar observations of auroral arcs, Proc. Symp. Multipoint Measurements of Magnetospheric Processes, COSPAR, 8, 9(49), 1988a.

Carlson, H. C., Jr., R. A. Heelis, E. J. Weber, and J. R. Sharber, Coherent mesoscale convection patterns during northward interplanetary magnetic field, J. Geophys. Res., 93, 14501, 1988b. 
Costa, E., P. F. Fougere, and S. BAsu, Cross-correlation analysis and interpretation of spaced-receiver measurements, Radio Sci., 23, 141, 1988.

FEDOR, L. S., A statistical approach to the determination of three-dimensional ionospheric drifts, J. Geophys. Res., 72, 5401, 1967.

Hairston, M. R, and R. A. HeElis, Determination of the ionospheric convection pattern from DMSP data, SPI Conference Proceedings and Reprint Series, 8, 391, 1989.

Keskinen, M. J., H. G. Mitchell, J. A. Fedder, P. Satyanarayana, S. T. Zalesak, and J. D. Huba, Nonlinear evolution of the Kelvin-Helmholtz instability in the high-latitude ionosphere, J. Geophys. Res., 93, $137,1988$.

Tsunoda, R. T., High-latitude F-region irregularities: A review and synthesis, Rev. Geophys., 26, 719-760, 1988.

Valladares, C. E. and H. C. Carl.son, Jr., The electrodynamic, thermal, and energetic character of intense stable sun-aligned arcs in the polar cap, submitted to J. Geophys. Res., 1990.

VickREY, J. F. and M. C. Kelley, The effects of a conducting $E$ layer on a classical $F$ region cross-field plasma diffusion, J. Geophys. Res., 87, 4461, 1982.

Weber, E. J., J. Buchau, J. G. Moore, J. R. Sharber, R. C. Livingston, J. D. Winningham, and B. W. ReINISCH, $F$ layer ionization patches in the polar cap, J. Geophys. Res., 89, 1683-1694, 1984.

Weber, E. J., J. A. Klobuchar, J. Buchau, H. C. Carlson, Jr., R. C. Livingston, O. de la Beaujardiere, M. McCready, J. G. Moore, and G. J. Bishop, Polar cap $F$ layer patches: Structure and Dynamics, $J$. Geophys. Res., 91, 12121-12129, 1986.

Weber, E. J., M. C. Kelley, J. O. Ballenthin, S. Basu, H. C. Carlson, J. R. Fleischman, D. A. Hardy, N. C. Maynard, R. F. Pfaff, P. Rodriguez, R. E. Sheehan, and M. Smiddy, Rocket measurements within a polar cap arc: Plasma, particle, and electric circuit parameters, J. Geophys. Res., 94, 6692, 1989. 